\title{
La généralisation des enquêtes judiciaires et la construction du pouvoir princier dans le duché de Bourgogne (XII ${ }^{\mathrm{e}}$-XIII ${ }^{\mathrm{e}}$ siècles)
}

\section{Armando Torres Fauaz}

\section{OpenEdition}

1 Journals

\section{Édition électronique}

URL : https://journals.openedition.org/cem/12028

DOI : $10.4000 /$ cem. 12028

ISSN : 1954-3093

\section{Éditeur}

Centre d'études médiévales Saint-Germain d'Auxerre

\section{Édition imprimée}

Pagination : 369-372

ISSN : 1623-5770

Référence électronique

Armando Torres Fauaz, "La généralisation des enquêtes judiciaires et la construction du pouvoir princier dans le duché de Bourgogne (x॥l - -xII ${ }^{\mathrm{e}}$ siècles) », Bulletin du centre d'études médiévales d'Auxerre I BUCEMA [En ligne], 15 | 2011, mis en ligne le 24 août 2011, consulté le 22 septembre 2022. URL: http://journals.openedition.org/cem/12028 ; DOI : https://doi.org/10.4000/cem.12028

Ce document a été généré automatiquement le 22 septembre 2022.

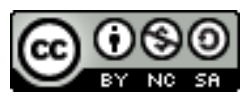

Creative Commons - Attribution - Pas d'Utilisation Commerciale - Partage dans les Mêmes Conditions 4.0 International - CC BY-NC-SA 4.0

https://creativecommons.org/licenses/by-nc-sa/4.0/ 


\title{
La généralisation des enquêtes judiciaires et la construction $\mathrm{du}$ pouvoir princier dans le duché de Bourgogne ( $\mathrm{XII}^{\mathrm{e}}$-XIII ${ }^{\mathrm{e}}$ siècles)
}

\author{
Armando Torres Fauaz
}

1 Ce projet est une étude historique du processus de généralisation de l'enquête judiciaire dans le duché de Bourgogne pendant les XII et XIII ${ }^{e}$ siècles. À partir de l'analyse des actes de la pratique et de la documentation ducale et papale, on étudiera comment les cours juridiques laïques et ecclésiastiques se sont adaptées aux transformations et innovations juridiques et procédurales à partir du XII ${ }^{\mathrm{e}}$ siècle, et surtout au XIII ${ }^{\mathrm{e}}$ siècle. Cette recherche s'articule autour de deux perspectives, que l'on ne doit pas considérer séparément. Il s'agit, d'une part, de l'analyse des procédures et leurs liens avec le droit savant, et, d'autre part, des rapports des enquêtes judiciaires avec la construction du pouvoir ducal pendant cette période.

\section{La perspective juridique}

2 La première de ces perspectives touche principalement la procédure des enquêtes judiciaires en Bourgogne. Il s'agit de reconnaître leurs rapports avec la tradition carolingienne survivante dans la pratique royale française et avec les modèles savants portés par les innovations canoniques du IV ${ }^{\mathrm{e}}$ concile de Latran.

3 Il importe de faire la lumière sur le fait, que, même si la tradition carolingienne, autant laïque que synodale, offre un modèle pour les enquêtes judiciaires et, surtout, administratives ${ }^{1}$, l'enquête, en tant que procédure pour juger les causes civiles, atteint le sommet de sa diffusion au XIII ${ }^{e}$ siècle. Certes, d'après la tradition savante, la procédure accusatoire subit déjà des réformes naissantes au XII siècle $^{2}$. Néanmoins, c'est seulement après la réformedu IV ${ }^{\mathrm{e}}$ concile de Latran, dans la clause Qualiter et 
quando du pape Innocent III, que l'enquête judiciaire se transforme. De cette manière, elle devient la procédure la plus sollicitée lorsqu'il s'agit de juger les causes civiles et criminelles durant les siècles postérieurs ${ }^{3}$.

4 Nous chercherons à voir comment cette innovation procédurale dans le duché de Bourgogne s'est traduite, quels en furent les foyers d'introduction, et quels modèles ont influencé précisément la forme à travers laquelle elle fut mise en place.

5 En premier lieu, il est nécessaire d'identifier, à travers l'analyse des actes de la pratique, les caractéristiques de la procédure d'enquête judiciaire au cours des $\mathrm{XII}^{\mathrm{e}}$ et XIII ${ }^{\mathrm{e}}$ siècles. Deux éléments sont observés. Premièrement, comment les enquêtes de cette époque reproduisent les dispositions paradigmatiques des enquêtes carolingiennes. Ensuite, dans quelle mesure la procédure de ces enquêtes conserve des ressemblances avec les procédures de règlement des conflits typiques aux $\mathrm{XI}^{\mathrm{e}}$ et $\mathrm{XII}^{\mathrm{e}}$ siècles. De cette manière, dans les causes civiles et les enquêtes ducales, il faut étudier le rôle des témoins, la nature des preuves admises dans la procédure, l'autorité qui met en place l'enquête, la façon par laquelle le conflit est réglé (d'après les actes) et le déroulement des interrogatoires.

Puis, il importe d'effectuer une analyse de la pénétration des dispositions procédurales canoniques, notamment à travers les causes jugées dans les cours ecclésiastiques et les interventions pontificales, spécifiquement lorsque le pape demande la réalisation des enquêtes dans le territoire bourguignon.

\section{La perspective « historico-anthropologique »}

7 La seconde perspective, à partir de laquelle on peut étudier la question, touche la compréhension des rapports entre ce que nous appelons la généralisation de la pratique de l'enquête et le processus de renforcement et d'expansion du pouvoir ducal en Bourgogne.

Cette expansion se consolide surtout grâce à une opposition au pouvoir royal et à celui des abbayes dans le sud du duché - d'où la guerre de Vergy, 1186 -; tandis que dans l'ouest et le nord, ce sont les seigneuries épiscopales de Langres et Autun qui sont en conflit avec le duc. Traditionnellement, on a cru que les ducs et leur entourage avaient réussi à surmonter ces contraintes et à étendre leur pouvoir dans le duché durant les règnes d'Eudes III, d'Hugues IV et de Robert II ${ }^{4}$. En réalité, il semble que cette expansion ne fut ni exclusive, ni principalement foncière. Il s'est plutôt agi de tisser un complexe réseau social et une sphère d'influence considérable avec les sires locaux, aussi bien laïcs qu'ecclésiastiques. De la même manière, d'autres moyens sont utilisés par les ducs, que nous pouvons qualifier de véritable appareil administratif. On pense aux "gardes » de monastères et de villes, aux hautes et basses «justices » dans les terroirs ou à l'imposition d'une division territoriale en bailliages.

Dans cette construction d'un appareil administratif, ce projet se concentre sur l'administration de la justice et sur l'extension effective du pouvoir ducal. Ainsi donc, en étudiant la documentation judiciaire, laïque et ecclésiastique, on analyse le rôle et la portée des cours laïques, des officiers ducaux et de la cour du duc. Ceci mène à l'étude de l'enquête proprement dite et à l'analyse de son rôle dans le processus d'expansion du pouvoir ducal ${ }^{5}$. 
10 Il importe d'expliquer que, en raison de la fondation d'une chapelle ducale, liée à l'église Saint-Étienne de Dijon, la documentation bourguignonne subit une importante mutation dès la fin du XII ${ }^{\mathrm{e}}$ siècle. On voit, alors, apparaître, pour la première fois, des figures issues de l'administration de la justice, qui ont été très rarement mentionnées jusque-là. Ainsi, les figures du sénéchal, du maréchal, du chambellan et du chancelier commencent à figurer dans les actes, entre la fin du XII ${ }^{\mathrm{e}}$ et le début du XIII ${ }^{\mathrm{e}}$ siècle.

11 Néanmoins, c'est l'apparition de trois autres figures dans la documentation de cette époque, qui révèle le changement le plus intéressant :

- tout d'abord, nous voyons de nouvelles mentions d'officiers ducaux, que Jean Richard et Ernest Petit ont identifiés comme des ministeriales, servientes, ministri ou, simplement, clientes ${ }^{6}$. Ceux-ci sont chargés, dès la fin du XII ${ }^{e}$ siècle, quand le pouvoir foncier du duc est encore limité, de rendre la justice et de lever les charges foncières. On les aperçoit à Dijon, à Autun, à Argilly, à Beaune et à Châtillon, où ils sont apparemment chargés de mener des enquêtes. Toutefois ce sont plutôt des enquêtes administratives, voire foncières, destinées à déterminer les droits du duc dans les terroirs;

- ensuite, au début du XIII ${ }^{\mathrm{e}}$ siècle, J. Richard voit une deuxième figure sous le nom de conciliarius,mais que E. Petit identifie tout au long du XIII ${ }^{\mathrm{e}}$ siècle comme iurisperitus. Cette figure est un lien substantiel pour comprendre les rapports entre la justice ducale et le droit savant. Ce type de conseiller est signalé par Jean Richard dès 1219 , mais le premier qu'il arrive à identifier est Lambertus de Rovra, au service du duc dès 1238-1240. En reconstruisant son histoire, il découvre qu'il était avocat et au service de plusieurs établissements religieux : notamment Saint-Étienne de Dijon la chapelle ducale liée au chapitre de Saint Bénigne de Dijon, Luxeuil, Notre-Dame de Beaune et l'abbaye de Cîteaux. Il en est de même avec le deuxième conseiller identifié, Jean de Blanot, professeur à Bologne en 1250, où il rédige un texte célèbre sous le titre De Actionibus ${ }^{7}$. En tenant compte de ces officiers, on peut conclure que le duc, ou au moins sa cour ou sa chancellerie, a un conseiller de droit savant à partir du début du XIII' siècle. Pourtant, si l'on fait une recherche simple dans les $\mathrm{CMBA}^{8}$, on trouve peu d'occurrences pour le mot jurisperitus. Toutefois, on remarque que ces références commencent dès 1198 et vont jusqu'à 1300. Les documents étant principalement de nature ecclésiastique, ils font sûrement référence à des conseillers juridiques des établissements ecclésiastiques - l'abbaye de Sainte-Colombe de Sens, pour le premier document ${ }^{9}$. Dans le document de $1214^{10}$, il s'agit, en effet, d'une enquête de type épiscopal, où trois membres du chapitre d'Auxerre sont venus à l'abbaye de Saint Martin d'Autun au sujet de la possession des dîmes. On peut donc supposer que la figure du jurisperitus ducal provient surtout de l'usage ecclésiastique. Ici, le lien entre le droit savant et la justice ducale est clairement marqué par l'administration de la justice ecclésiastique, mais également avec l'enquête.

12 Enfin, une troisième figure administrative apparaît dans cette documentation: le mandatum ducal. Jean Richard, qui évoque très laconiquement cette figure, pense qu'après 1246 cet officier est chargé d'aller dans les communautés pour faire valoir les droits et les prérogatives ducaux. À travers l'exercice des enquêtes ducales, cette figure, semble-t-il, tisse un réseau de pouvoir administratif dans les territoires qui composent le duché. Il rend la justice du duc et représente son autorité.

13 Ces trois figures permettent de lier l'enquête, foncière et judiciaire, avec la construction d'un appareil administratif ducal. Néanmoins, les spécificités des rapports entre la généralisation de cette pratique, l'expansion et le renforcement du pouvoir ducal restent encore à explorer. Pour mettre cette question au jour, il faut, d'une part, comprendre le processus de la généralisation de cette pratique, et, d'autre part, savoir 
quel rôle ont joué les enquêtes dans les rapports de force entre les ducs et les sires locaux, voire les comtes, les évêques, les abbés et les seigneurs. De la même manière, nous devons vérifier si l'enquête joue un rôle dans la politique locale et de quelle façon sa mise en pratique affecte la dynamique interne de rapports de force dans le duché. Autrement dit, il convient d'analyser les enjeux relatifs à leur usage.

\section{Objectifs généraux}

Par conséquent, en fonction de ce que nous venons d'exposer, les objectifs de ce projet peuvent être résumés de la manière suivante :

- étudier le processus de généralisation de la pratique inquisitoire dans le duché de Bourgogne, aux XII et XIII ${ }^{e}$ siècles, tant dans les cours laïques que dans les cours ecclésiastiques ;

- étudier le rôle des enquêtes dans la construction d'un appareil administratif ducal et dans l'expansion du pouvoir des ducs sur le territoire bourguignon ;

- étudier l'impact que la mise en place de la pratique inquisitoire a sur les relations de pouvoir à l'intérieur du duché ainsi qu'entre l'office ducal, le roi de France, et les institutions ecclésiastiques ;

- étudier les transformations dans la procédure de l'enquête judiciaire en Bourgogne aux XII et XIII siècles et la relation de ce phénomène avec les formes carolingiennes et les innovations canoniques à la suite du IV concile de Latran.

\section{NOTES}

1. B. LEMESLE, «Premiers jalons et mise en place d'une procédure d'enquête dans la région angevine ( $\mathrm{XI}^{\mathrm{e}}-\mathrm{XIII}{ }^{\mathrm{e}}$ siècle) », in $\mathrm{B}$. LEMESLE (dir.), La preuve en justice. De l'Antiquité à nos jours, Rennes, 2003, p. 69-72.

2. R. M. FRAHER, «The theoretical justification for the new criminal law of the high middle ages: "Rei publicae interest, ne crimina remaneant impunita"», in University of Illinois Law Review, 3 (1984), p. 577-595.

3. R. M. FRAHER, «IV Lateran's revolution in criminal procedure : The birth of inquisitio, the end of ordeals and Innocent III's vision of ecclesiastical politics ", in Studia in honorem eminentissimi cardinalis Alphonsi M. Stickler, Rome, 1992, p. 101.

4. J. RICHARD, Les ducs de Bourgogne et la formation du duché du XI ${ }^{e}$ au XIV siècle,Paris, 1954, p. 190-235.

5. Il est intéressant de considérer une des versions de la charte de commune de Dijon, où il est spécifié que les juges communaux doivent faire appel à une enquête s'ils doutent de la crédibilité des témoins produits pour une cause, cf. archives départementales de la Côte-d'Or, B 10423, fol. $357 \mathrm{v}^{\mathrm{o}}$.

6. J. RICHARD, Les ducs..., op. cit., p. 401-435. E. PETIT, Histoire des ducs de Bourgogne de la race capétienne,Paris, 1885,t. 4, pièces n 6397-6398.

7. Édité partiellement dans J. ACHER, « Notes sur le droit savant au Moyen Âge », La nouvelle revue historique de droit, 30 (1906), p. 138-178. Cf. J. RICHARD, « Les exemples Bourguignons dans le traité 
des hommages et des fiefs de Jean de Blanot ", Mémoires de la société pour l'histoire du droit et des institutions des anciens pays bourguignons, comtois et romands, 18 (1965), p. 107-112.

8. En ligne : http://www.artehis-cbma.eu/.

9. M. QUANTIN, Cartulaire général de l'Yonne, t. 2, Auxerre, 1860, p. 486.

10. M. QUANTIN, Recueil de pièces pour faire suite au Cartulaire général de l'Yonne: XIII siècle, Auxerre, $1873, n^{\circ} 150$.

INDEX

Index géographique : France/Bourgogne

Mots-clés : enquête judiciaire, pouvoir princier 